\title{
Dynamics of cyanobacterial global metabolom in response to light and temperature variations
}

\author{
Damien Le Moigne ${ }^{1}$, Justine Demay ${ }^{1,2}$, Anita Reinhardt ${ }^{2}$, Cécile Bernard ${ }^{1}$, Sandra Kim Tiam ${ }^{1,3}$ and Benjamin Marie \\ $1, *$
}

1 UMR7245 MCAM MNHN-CNRS, Muséum National d'Histoire Naturelle, CP 39, 12 rue Buffon, F-75231 Paris Cedex 05, France.; damienlemoigne@gmail.com (DLM); justine.demay1@mnhn.fr (JD); cecile.bernard@mnhn.fr $(\mathrm{CB})$;

2 Thermes de Balaruc-Les-Bains, 1 Rue du Mont Saint-Clair BP 45, 34540 Balaruc-Les-Bains, France; anita.reinhardt@thermesbalaruc.com

3 Université de Lyon, Université Lyon 1, CNRS, UMR5557, Ecologie Microbienne, INRA, UMR 1418, 43 bd du 11 novembre 1918, F-69622 Villeurbanne, France; sandra.kim-tiam-fook-chong@univ-lyon1.fr (SKT)

* Correspondence: benjamin.marie@mnhn.fr (BM); Tel.: +33-1-40-79-32-12

\begin{abstract}
Cyanobacteria are microorganisms able to adapt to a wide variety of environmental conditions and abiotic stresses. They produce a very large number of metabolites that can participate in the adaptation of cyanobacteria to a large range of resources such as light, temperature, or nutrient. The metabolites variation is one way to understand the physiological status and adaptation of cells. In this study, we aim to understand how the diversity and the dynamics of the whole metabolome is dependent of the growth phases and under control of abiotic factors (e.g. light intensity and temperature). The cyanobacteria Aliinostoc sp. PMC 882.14 was selected for its large number of biosynthetic gene clusters. Metabolomes were analyzed by using mass spectrometry (qTOF-MS/MS) combined with untargeted analysis to investigate the metabolite dynamics. Significant variations were characterized between exponential and stationary phases, whatever the culture conditions ("control", "higher light", or "higher temperature"). "Higher light" and "higher temperature" favored the synthesis of metabolites belonging to the same molecular families. Among highly regulated metabolites, we observe the presence of mycosporine-like amino acids (MAAs), and various variants of somamides, microginins, and microviridins. Through Aliinostoc sp. PMC 882.14, this study shows the importance of knowing the physiological state of cyanobacteria for comparative global metabolomics and questions the regulation processes involve into metabolite families production. Our results also open up new perspectives in the context of the production of targeted bioactive metabolites.
\end{abstract}

Keywords: cyanobacteria; metabolomics; high-resolution mass spectrometry; secondary metabolite induction; culture conditions

\section{Introduction}

Cyanobacteria are gram-negative photosynthetic prokaryotes that can be found in all environments and habitats including extreme conditions. They play an essential role in the functioning of various ecosystems since they have a key role in primary production by experiencing oxygenic photosynthesis processes, containing both chlorophyll $a$ and other more specific pigments such as phycocyanin or phycoerythrin [1].

Cyanobacteria produce a wide variety of bioactive metabolites, as more than 1,100 metabolites have been described to date [2]. This variety of metabolites seems to support the remarkable ecological capacities of cyanobacteria [3]. As a consequence, cyanobacteria are organisms of choice for researching valuable bioactive molecules and various scientific fields aims to explore their properties, such as pharmacology, food industry or biotechnology $[4,5]$. They also present promising applications in the field of cosmetics thanks to 
their production of various molecules with photo-protective, antioxidant or anti-inflammatory properties (e.g. carotenoids, mycosporine-like amino acids (MAAs), scytonemins, etc.) $[5-7]$.

A large portion and diversity of cyanobacterial secondary metabolites are peptides or exhibit peptide structures [8], being synthesized by specific enzymatic biosynthetic machineries [2], through either ribosomal (ribosomally synthesized and post-translationally modified peptides - RiPPs) or non-ribosomal (non-ribosomal peptide synthase, NRPS, or polyketide synthase, PKS) pathways [9]. However, many other secondary metabolites can present different structure organization and belong to other chemical classes such as alkaloids, terpenes, polysaccharides or even lipids [10]. Nevertheless, the vast majority of the biological roles of these cyanobacterial secondary metabolites remain unknown [11], although certain hypotheses regarding their respective potential biological functions (e.g. allelopathy, defense, parasitism/symbiosis, chemical communication, etc.) have been proposed [12].

So far, a large number of studies are available on targeted molecules producing regarding the physiological status and/or growth phases with in vitro experimentations approaches [13]. However, only few studies focused on the whole cyanobacterial metabolome and its dynamics over time and under different culture conditions [14]. Interestingly, a pilot study performed on diatoms [15] demonstrated a clear specificity of the metabolic profiles in regard to the different growth phases (exponential, stationary and senescence phases).

Several factors are supposed to be able to influence the growth of cyanobacteria, including, temperature, light or nutrient availabilities. Among them, authors showed that temperature $\left(25^{\circ} \mathrm{C}\right)$ improved the growth of the bloom-forming Microcystis strains while lack of light or nutrients negatively impacted the growth of cyanobacteria [16-18]. Beyond its effects on cyanobacterial growth, light has also been described as a key factor driving specific metabolites production. As an example, cyanobacteria are able to set up photoprotection mechanisms with the production of molecules that absorb UV-radiation (e.g. MAAs; [19]) or minimize the production of reactive oxygen species and protect DNA thanks to specific UV-inducible defenses (e.g. scytonemins; [6]).

In the present study, we investigated the whole metabolite production of the cyanobacteria Aliinostoc sp. PMC 882.14, isolated from biofilms of thermal mud of Balaruc-lesBains (France) [20]. Aliinostoc sp. PMC 882.14 has been selected for exhibiting a large number of biosynthetic gene clusters (BGCs) (supplementary table S1) able to produce a wide variety of metabolites valuable in the cosmetic or pharmaceutical sectors, such as MAAs (e.g. antioxidant, anti-UV properties) or microginin, symplostatin and dolastatin (e.g. cytotoxic activities) (supplementary figure S1) [21].

This work aims to describe the diversity of metabolites produced by the strain Aliinostoc sp. PMC 882.14 and to study the importance of culture conditions on the metabolome content using qualitative and quantitative untargeted metabolomic approaches. We focus on the effect of light and temperature on the composition of endo- and the exometabolites along cyanobacteria growth. We hypothesized that the increase of light intensity and temperature increases the production of specific metabolites allowing adaptation of cyanobacteria to the variation of these two abiotic factors. We characterized that some of them were especially relevant for cosmetic or pharmaceutical application. 


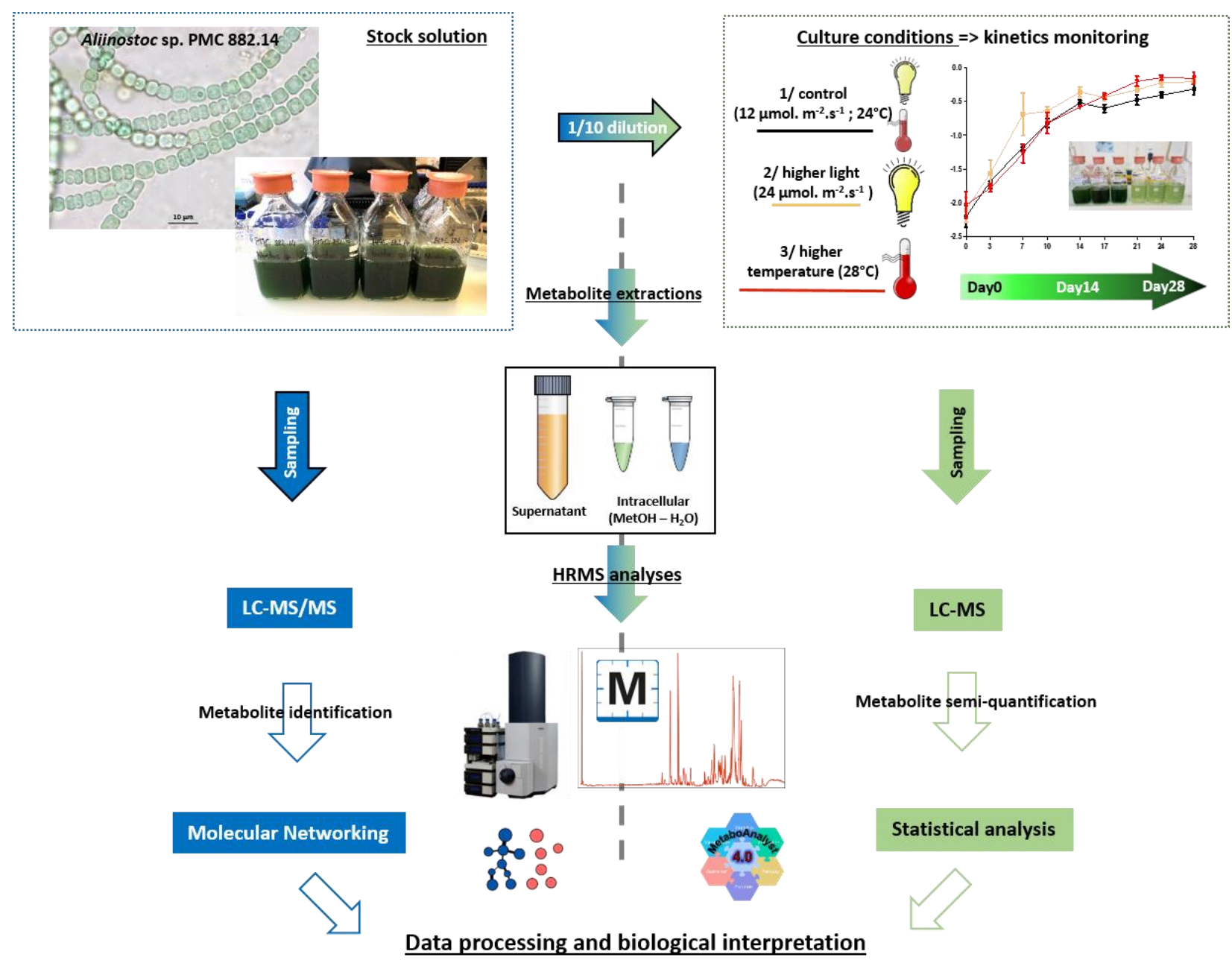

Figure 1. Graphical abstract of the analytical pipeline performed in this study

\section{Results}

\subsection{Extraction compartment specificity and metabolite diversity of Aliinostoc sp. PMC 882.14}

The three fractions (intracellular extracted with water (IW) or methanol (IM), and the extracellular supernatant (E)) retrieved from the pre-culture of the strain Aliinostoc sp. PMC 882.14 (in control condition) were first investigated in both positive and negative ionization MS mode. Taken all three fractions together (IW, IM and E), 1,971 different ions were observed in positive mode (79\% of total ions detected), while 518 ions (20\% of the total ions detected) were observed in negative mode, with only 48 ions commonly detected in both positive and negative ionization modes. Accordingly, we assume to focus rationally our investigation on metabolome dynamics to the MS analysis performed on the positive mode solely.

Figure 2 exhibits the respective composition (in relative intensity) of the ions analyzed in LC-MS (using merged positive and negative mode dataset) for the three fractions analyzed (IW, IM and E). Figure 2a shows that the three extraction fractions have a very different metabolic composition, especially the extracellular fraction (E) that present a very different metabolic composition from the two intracellular fractions (IW and IM). These observations were also illustrated on the individual plot of the principal component analysis (PCA) (Figure 2b) and the Venn diagram (Figure 2c), indicating that similar numbers of analytes (chemical entities regrouping all observed charge states and adduct forms) were observed in the three fractions (totals of 1596, 1789 and 1693 analytes detected in the E, IM and IW fractions, respectively), 848 analytes (34.7\%) being present in the three extracted fractions. On the other hand, these fractions contain 413, 191 and 45 ions that were found exclusively in the E, IW and IM fractions, respectively, representing 16.9\%, 
$7.8 \%$ and $1.8 \%$ of the total analytes detected. Noticeably, a greater number of common analytes were observed between IW and IM (609 analytes, 24.9\%), endorsing the fact that both being obtained from the intracellular compartment, when IM and E fractions share only 194 analytes (7.9\%) and the IW and E only 141 ions (5.8\%).

Further fragmentation experiments were performed on these three cellular fractions using LC-MS/MS positive and negative ionization modes. Out of the total of the 2,441 analytes detected with simple LC-MS experiments, 1,530 analytes could have been analyzed by LC-MS/MS. These MS/MS fragmentation data were used for metabolite annotation (supplementary table S2) and molecular network construction (Figure 2d). In this network, each analyte is represented by a node which diameter depends on its respective total ion intensity, the color area indicating the corresponding portion of the cellular fractions in which it has been detected. Clouds group together ions that present similar MS/MS fragmentation profiles according to GNPS/MetGem algorithm. The most important clusters are constituted by molecules isolated in the supernatant and comprise molecules possessing $\mathrm{COOH}$ acid groups (cepteic, roccellic, glutamic acids). Remarkably, this $\mathrm{E}$ fraction also contains various fatty acids (roccellic acid) and amino derivatives of fatty acids (erucamide). On the other side, the intracellular metabolites that were isolated with water (polar, in blue) and methanol (non-polar, in green), comprise, respectively, hydrophilic compounds, presenting noticeable osidic residues such as nucleosides, maltopentose and glycan tri-saccharides, or more hydrophobic molecules (mainly lipids), such as glycerolipids or erythro sphingosines. Other components presenting more intermediary hydrophobicity were retrieved in both IM and IW fractions, and remarkably not in the supernatant, comprising, among others, dolastatins, microginins and various amino acids or peptides. 


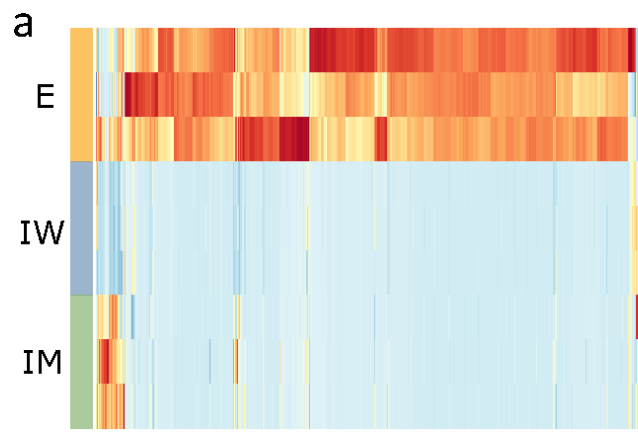

b

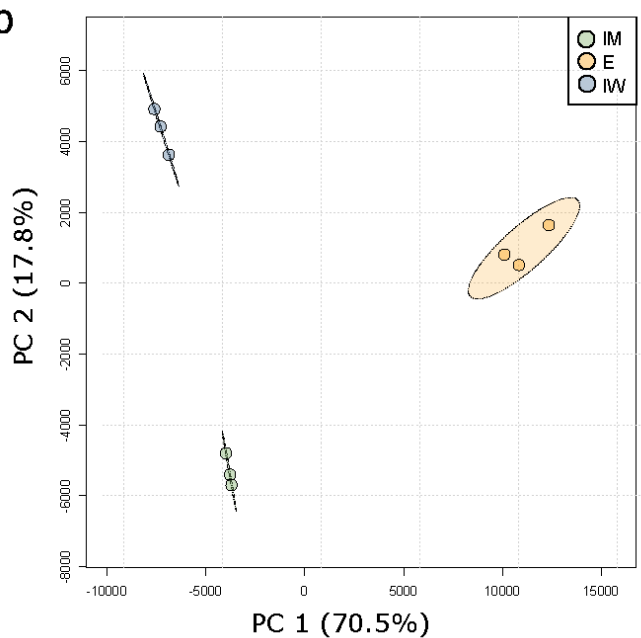

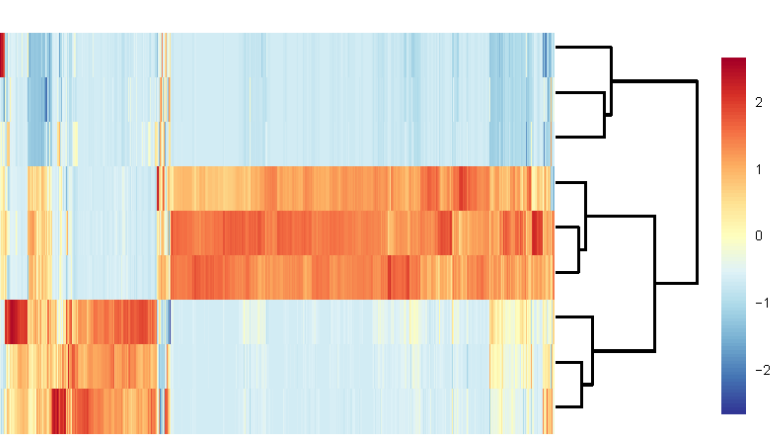

C

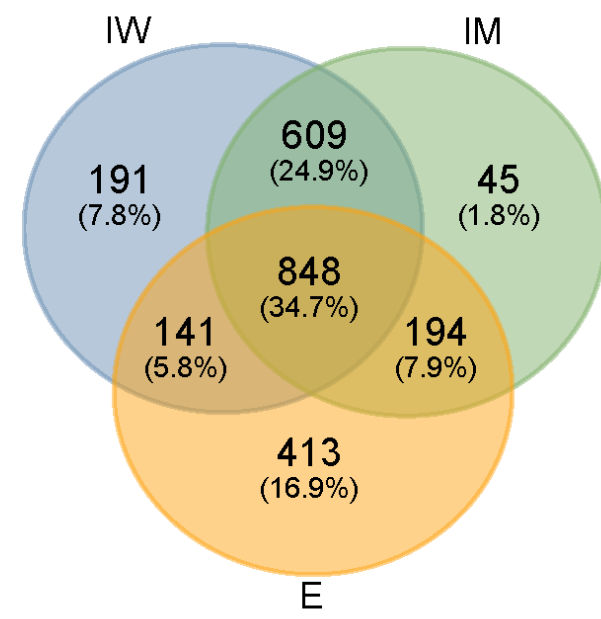

d
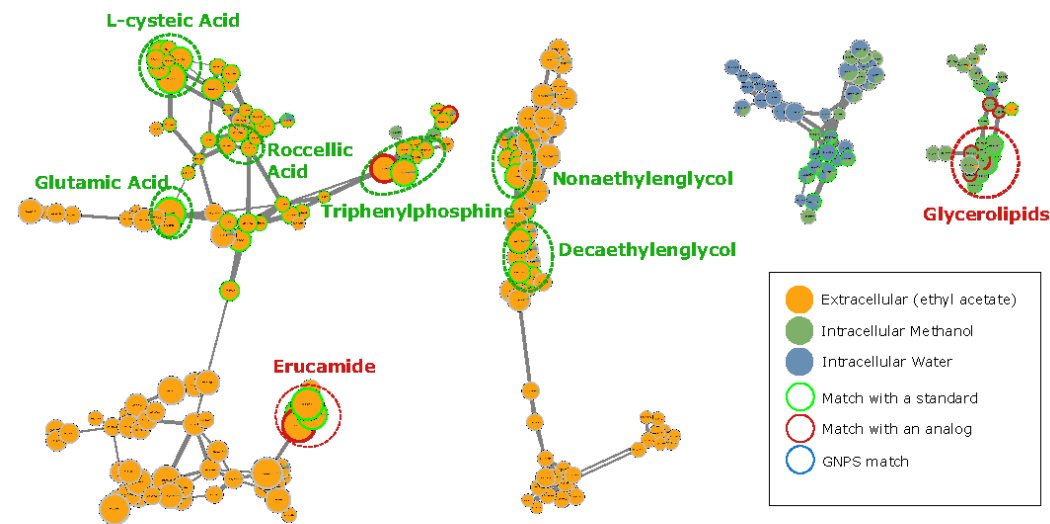

Glycerolipids

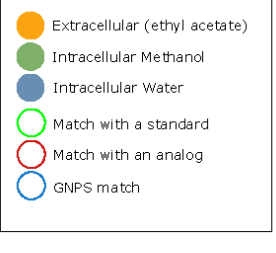

Glycerolipids

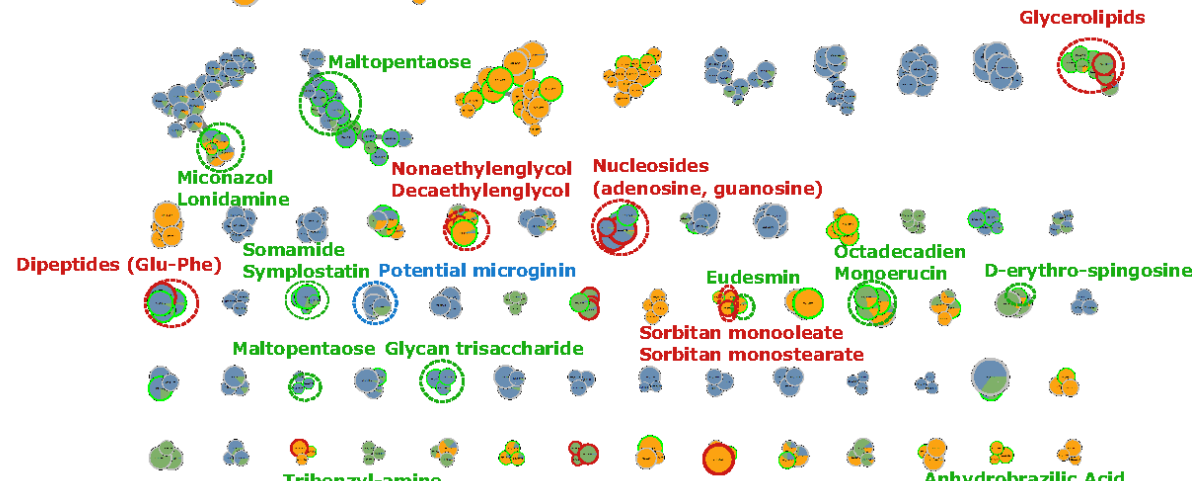

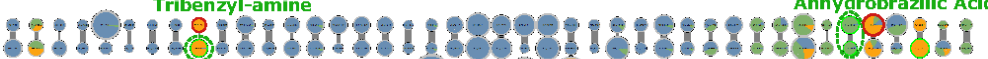

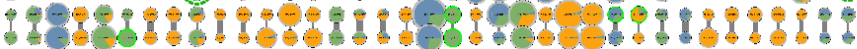

Figure 2. Metabolic composition of the three extraction fractions (triplicates) illustrated by a) a heatmap with hierarchical classification, b) a Principal Component Analysis, c) a Venn diagram and d) molecular network representation of the molecules detected in each one of the three extraction fractions. E, extracellular; IW, intracellular-water; IM, intracellular-methanol. On molecular network, the dots represented the different ions analyzed in autoMS/MS (merged positive and negative mode dataset). The colors of the dots corresponded to the fraction in which the analytes have been detected. The annotations were made with the MetGem 1.2.1 software (red and green dotted circles) or with the GNPS web tool (blue dotted circle) using public spectral databases. Only clusters of at least two nodes are represented. 
2.2. Dynamics of metabolite production under control, "higher light" and "higher temperature" conditions

2.2.1. Influence of growth on metabolite dynamics

During the 28 days of PMC 882.14 Aliinostoc sp. culture, the $750 \mathrm{~nm}$ absorbance (Figure 3), the chlorophyll $a$ concentration and the cell abundance (supplementary figure S2) showed a characteristic growing pattern with an exponential phase (from D0 to D14), then a stationary phase (after D14). Although, these two phases were being observed whatever the three culture conditions. The comparison of the different growth curves with those obtained under control conditions $\left(24^{\circ} \mathrm{C}, 12 \mu \mathrm{mol} . \mathrm{m}^{-2} \mathrm{~s}^{-1}\right)$ shows few significant variations with a higher growth of Aliinostoc exposed to "higher light" $\left(24 \mu \mathrm{mol} . \mathrm{m}^{-2} \cdot \mathrm{s}^{-1}\right)$ at D7 (exponential phase, pvalue $<0,001)$ and to "higher temperature" $\left(28^{\circ} \mathrm{C}\right)$ at D21 (stationary phase, pvalue $<0,05$ ).

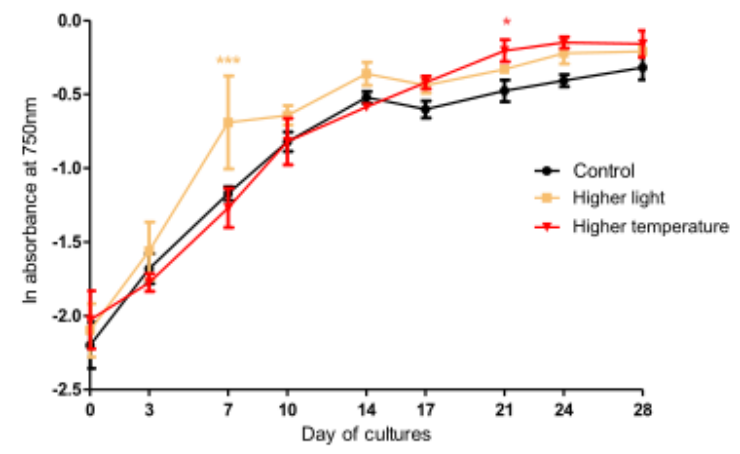

Figure 3. Cell growth curves of Aliinostoc sp. PMC 882.14 according to different culture conditions ("control" $24^{\circ} \mathrm{C} / 12 \mu \mathrm{mol} . \mathrm{m}^{-2} . \mathrm{s}^{-1}$, "higher light" $24 \mu \mathrm{mol} . \mathrm{m}^{-2} . \mathrm{s}^{-1}$, "higher temperature" $28^{\circ} \mathrm{C}$ ) according to the number of culture days considering the in vivo absorbance at $750 \mathrm{~nm}$ (mean $\pm \mathrm{SD}$ ). Significant differences between control and treatments ("higher light", "higher temperature") were assessed by the ANOVA ( $p<0,001$ for Higher light at D7 and $p<0,05$ for Higher temperature at D21) corrected with Bonferroni's test.

Regarding the intracellular metabolome (pooled IM and IW fractions), the variations of the global metabolome composition during the 28 days of culture have first been explored with un-supervised multivariate analysis (PCA, principal component analysis) as illustrated in Figure 4, for control condition only (Fig. 4a) or for all three experimental conditions (Fig. 4b). A progressive shift of the global metabolome among the duration of the culture was observed. Although the replicated measurements illustrate a reliable replication of the experiments, it also shows a progressive disparity increase between triplicates through the growing kinetic and/or under the different experimental conditions, in a lesser extent. On the other hand, the points corresponding to the first days of sampling (D0, D3 and D7) are very close to each other, suggesting that only little metabolic composition changes occur during the first seven days of the culture. Interestingly, the PCA analyses mostly discriminated the samples from D0 to D14 according to the component 1 (representing 58 and $45 \%$ of the total variance), when the component 2 (10 and $11 \%$ of the total variance) clearly discriminates samples from D14 to D28, suggesting that two distinguishable metabolome regulation events (metabolic phase $1=$ from D0 to D14 days and metabolic phase 2 = from D14 to D28) could be distinguished along this 28-day kinetics. 

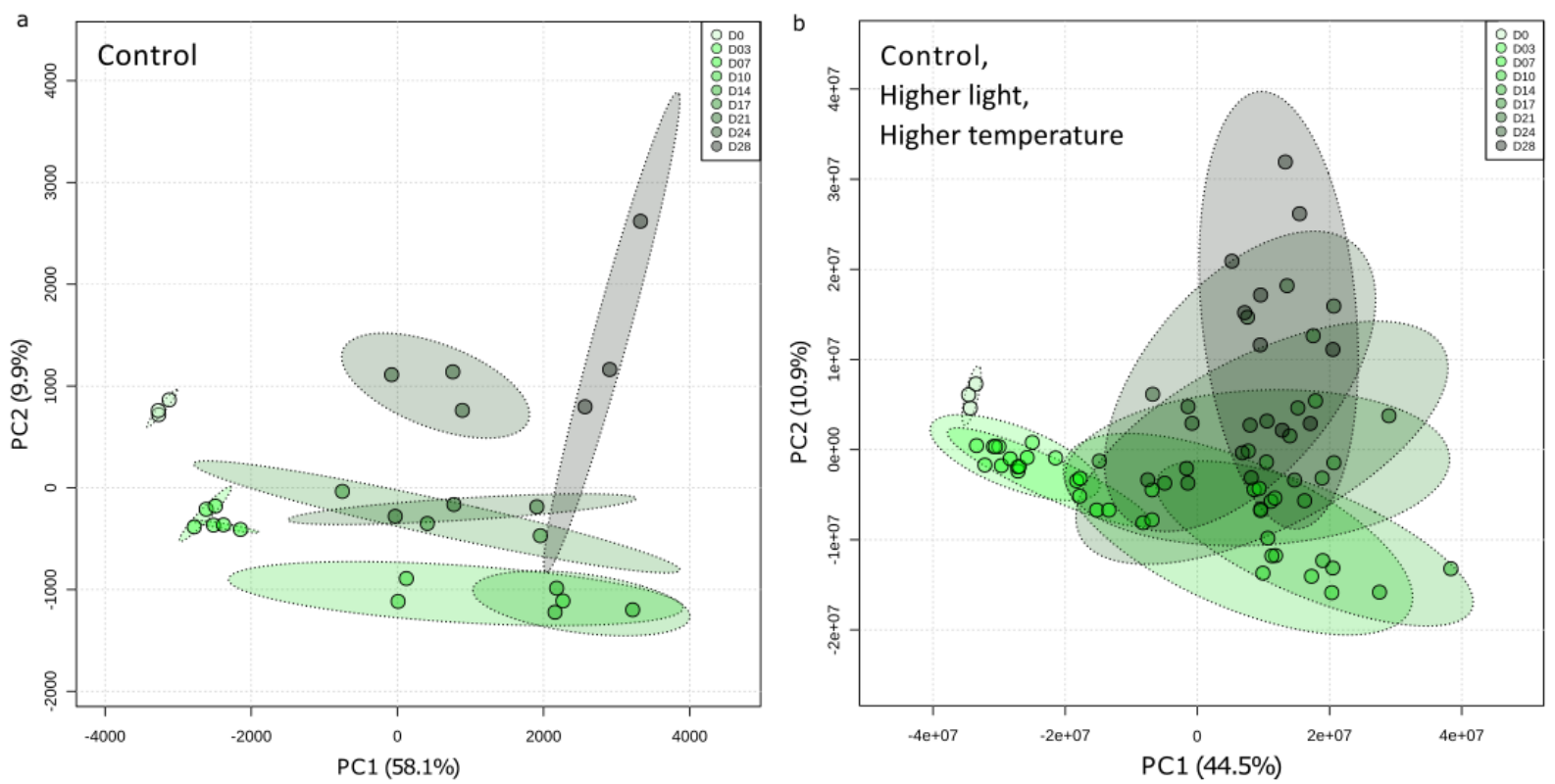

Figure 4. Principal Component Analysis (PCA) representing the evolution of the intracellular metabolic composition of Aliinostoc sp. PMC 882.14 as a function of number of culture days a) under the control condition only, or b) under control, "higher light" and "higher temperature" conditions. Each point represents a culture replicate. The green color gradient reflects the temporal scale of the cultures.

Supervised multivariate analysis (i.e. partial least square discriminate analysis, PLSDA) was then similarly conducted considering the days of sampling and represented on supplementary figure S4, for control samples only (Fig. S4a) and for the samples from the three experimental conditions (Fig. S4b). These individual plot PLS-DA representations exhibit very similar patterns than those observed previously for PCA (with relatively similar \%age of component 1-2 contributions too), and illustrate the good performance $\left(\mathrm{R}^{2} \mathrm{cu}-\right.$ mulative) and predictability $\left(\mathrm{Q}^{2}\right.$ cumulative $)$ scores. The list of the analytes that are contributing the most to the sample discrimination through the culture kinetics (variable of importance in the projection, VIP score $>2$ ) are represented on supplementary figures S4c and S4d. Interestingly, the list of variables that most discriminate samples during the kinetics are globally the same when considering the samples from the control condition only, or those from the three experimental conditions (bordered by red squares). Overall, and except for some rare examples, these intracellular metabolites globally present a net increase of their relative intracellular concentrations, suggesting that these compounds are accumulated within the cells during the culture kinetics (Figure 5, supplementary figure S4, supplementary figure S5 and supplementary figure S6). The observation of the individual analyte variations illustrates this global increase tendency over the time of culture, and also allows to distinguish three principal patterns comprising: $a$ ) one-step progressive increase at D10, as observed for some microginins, some dolastatins or the adenosine (Fig. 5a), b) two-step progressive increase at D3 and D17, as observed for microviridin 1724, for example (Fig. 5b), or c) a late, but important, increase occurring at D24, such as for the monoacylglycerol 16:1 (Fig. 5c). These observations suggest that various regulation processes controlling the intracellular content of those components may occur during the culture kinetics, depending to the molecule family and depending on their respective biosynthesis/consumption balance within the cyanobacteria. 


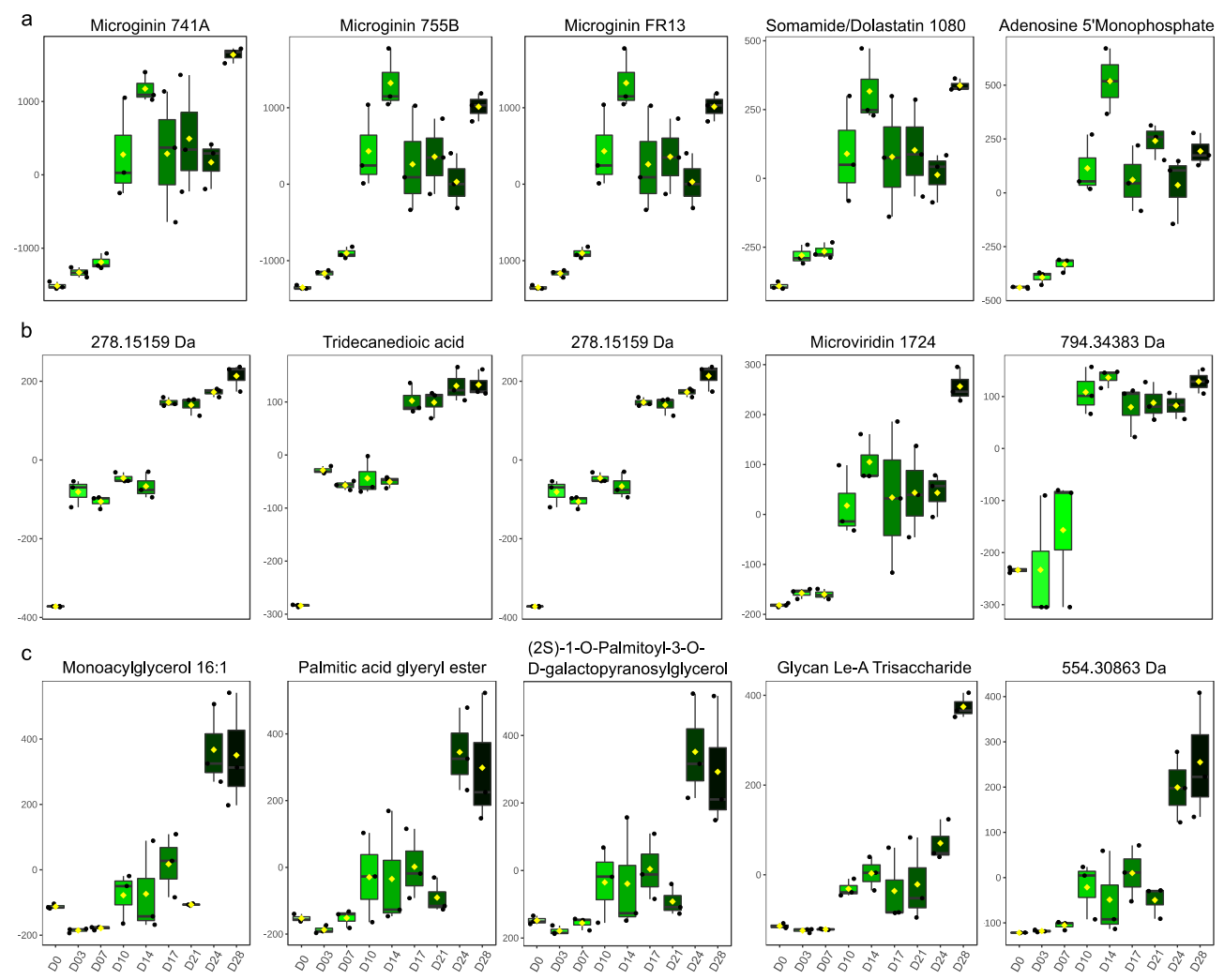

Figure 5. Box-plots representing a selection of 15 among the 38 intracellular molecules (exhibiting VIP scores > 2), which explain the most of the differences between the different days of culture. The three main patterns were illustrated with molecules whose relative intensity a) increases promptly at D14, b) increases by forming a transitional step between D10 and D21, c) increases mostly at the end of kinetics (D24 and D28).

\subsubsection{Influence of light and temperature variations on the metabolomic dynamics}

PCA analysis revealed that metabolite composition was under the control of culture conditions (Fig. S3). In order to identify the metabolites which relative quantity were the most modified by the two tested culture conditions, data were analyzed by a multivariate approach specifically dedicated to process time series investigation (MEBA, multivariate empirical bayes analysis). This approach highlights variables for which quantity variations over the time diverge the most between the different experimental conditions and was supported by two ways ANOVA investigations $(\mathrm{P}<0.001)$, revealing a cross-effect of the experimental conditions and the culture duration. The variation profiles of the metabolites presenting the best MEBA scores are illustrated in Figure 6 (Table S3).

Figure 6a shows metabolites whose intracellular relative concentrations is greater under "higher light" conditions when compared to the control. Overall, these metabolites follow a quite similar trend, characterized by an increase until D14, then a slight decrease. This comprises two microginins and two dolastatins, both presenting very similar patterns. For these components, this peak of maximal concentrations observed at D14 was also observed under control condition, but appears to be less marked. Interestingly, one of the metabolites belonging to the MAAs family, the shinorine (a potential photo-protector), exhibited a larger intracellular accumulation under "higher light" condition than in control, when almost no signal was detectable under the "higher temperature". Figure $6 \mathrm{~b}$ shows the metabolites whose intracellular content is greater under the "higher temperature" condition compared to the control. As exemplified for two microginins, two microviridins, as well as for adenosine monophosphate and an unknown metabolite of 238.95991 Da (neutral mass), we observed an initial relative concentration increase (up to 
D10) then a stabilization until D28. Quite different profiles were observed for the dipeptides Glu-Val, Glu-Cys as well as for the unknown 260.13684 Da metabolite that present important concentration increase after D21. It appears interesting to notice that, the maximum of the increase of the intracellular metabolite concentration seemed to be induced by "higher light" condition after 10-14 days of culture, then it decreased. Differently, the temperature-induced increases occurred latter during the kinetics (from D14), but appeared to be much more stable until D28.

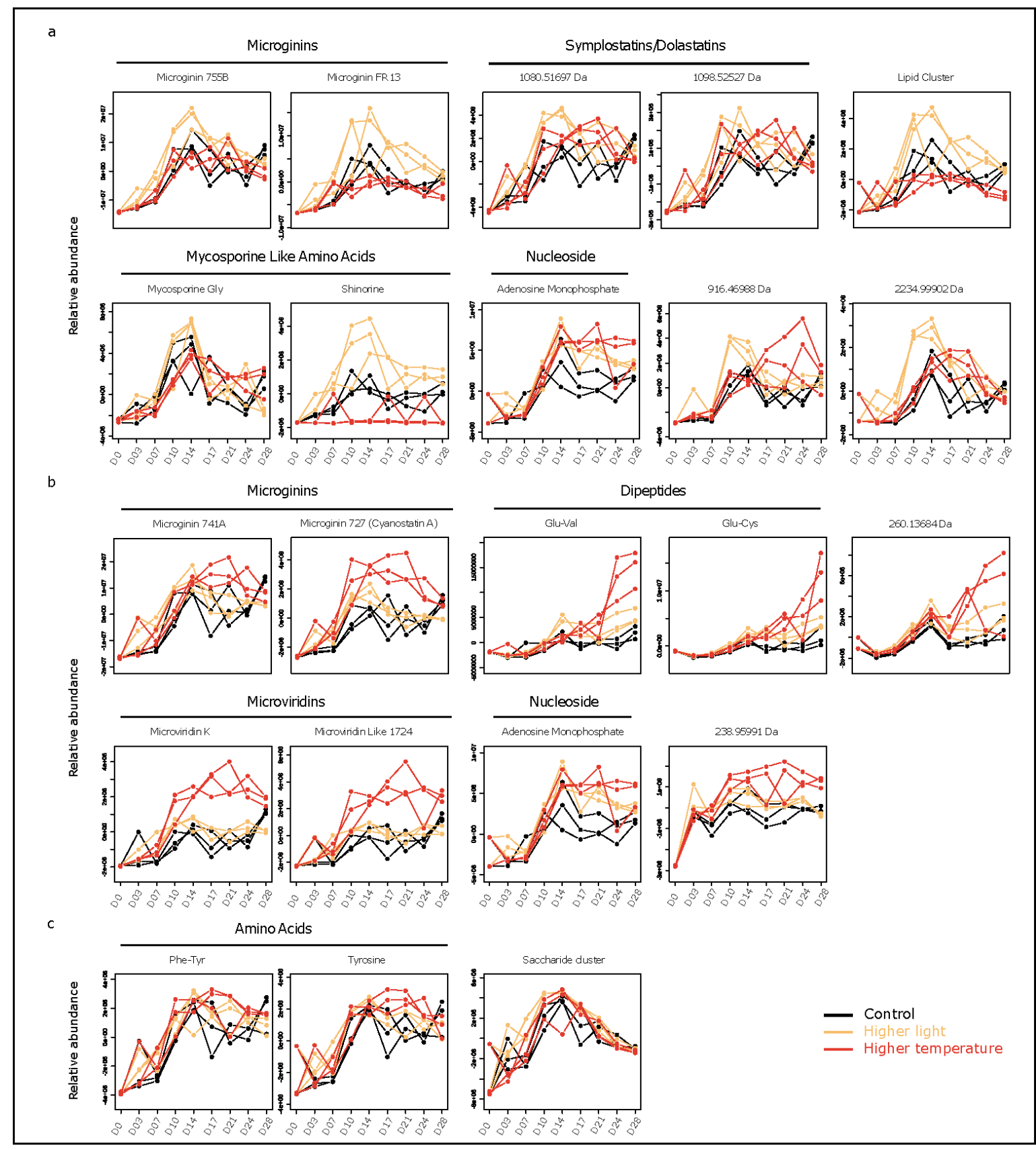

Figure 6. Relative abundance profiles of a selection of 22 analytes among the 48 presenting the best MEBA (Multivariate Empirical Bayes Analysis) classification scores, further confirmed by two ways ANOVA $(\mathrm{p}<0.001)$. a) Metabolites with a higher intracellular concentration in the "higher light" condition than in the control. b) Metabolites with a higher intracellular concentration in the "higher temperature" condition than in the control. c) Metabolites presenting more complex pattern of regulation when compared to the control. Each line representing a different replicated culture. 
On the other side, similar analyses were attempted for the extracellular analytes (Supplementary figure S7-S10) and lead to the observation that: i) the extracellular metabolome presented a net temporal variation along the time series, with important heterogeneity between replicates that appears through latter culture days (supplementary figure S7); ii) the experimental factors ("higher light" and "higher temperature") seemed to have a limited effect on the extracellular metabolome variation (supplementary figure S8); iii) the analytes that best discriminate sampling time were the same when considering only the control condition or all conditions together (supplementary figure S9); iv) these extracellular components corresponded mostly to unknown molecules presenting rather global increases or decreases during the time series (supplementary figure S10); v) no perceptible metabolite transfer from the intracellular compartment to the extracellular one could had been observed through the investigated time series covering both exponential and stationary growing phases.

\section{Discussion}

Analysis of the molecular network of metabolites of the Aliinostoc sp. PMC 882.14 indicates the presence of numerous generalist cellular metabolites such as dipeptides, nucleosides, or fatty acids, as well as, more specific molecules of cyanobacteria such as analogs of MAAs, somamides, microviridins and microginins.

Somamides are part of the class of cyclo-depsipeptides and have been isolated in particular from cyanobacteria of the genus Schizotrix and Lyngbya [22], but this family of molecules has been poorly described so far.

The Aliinostoc sp. PMC 882.14 also produces different variants of microginins (supplementary figure S1). These molecules are secondary metabolites of linear peptide structure produced by the NRPS/PKS hybrid biosynthetic pathway [23], that can lead to the formation of a large diversity of structural variants by a single strain [24]. To date, more than 90 variants of microginins have been already referenced in databases [25], isolated mainly from cyanobacteria of the genus Microcystis and Planktothrix but also from some cyanobacteria belonging to the genus Nostoc [26].

The monitoring of the metabolome variation through 28 days of culture under different experimental conditions indicates that time of culture was the main driver controlling the relative composition of both intra- and extra-cellular contents of Aliinostoc sp. PMC 882.14. This result was illustrated in Figure 4 since the samples corresponding to the different points of the time series were discriminated along component 1 for samples ranging from D0 to D14, then along component 2 for latter samples. This progressive temporal change of the intracellular metabolite contents across two distinguishable phases (D0-D14 and D14-D28) were in perfect correspondence with the growth phases (exponential phase from D0 to D14, then stationary phase) observed from the monitoring of the cell multiplication within the cultures (Figure 3 and supplementary figure S2).

A closer look at the evolution through the time series of the concentration of the variables responsible for the global metabolome variations (Figure 5; supplementary Figure S4-S5) reveals a global increase in their relative intracellular concentration. It also highlights the existence of even more subtle regulation processes probably implicating biosynthesis, accumulation and consumption events (Figure 5a-c). Interestingly, most metabolites belonging to the same molecule family present very similar variation patterns, claiming for the occurrence of homogeneous regulation processes through the different variants of each molecule families. While certain analytes, such as microginins exhibit a prompt and important increase of their relative concentration at the end of the exponential phase followed by a stabilization, the relative concentration of other molecules, such as microviridins, increases by forming a transitional step between D10 and D21. Differently, other metabolites present mainly a net increase during the late stationary phase (D24-D28).

The physical stimuli induced by "higher light" $\left(24 \mu \mathrm{mol} \cdot \mathrm{m}^{-2} \cdot \mathrm{s}^{-1}\right)$ or "higher temperature" $\left(28^{\circ} \mathrm{C}\right)$ conditions seems to have different cellular impacts since the accumulation of intracellular metabolites differs in term of quantity and kinetics between both conditions 
(Figure 6). In the present case, "higher light" induced more rapid metabolites variation than "higher temperature".

Indeed, the maximum of induction for microginin 755B and microginin FR13, symplostatin/dolastatin, MAAs (mycosporine-glycine and shinorine) as well as certain un-annotated metabolites under "higher light" conditions $\left(24 \mu \mathrm{mol} . \mathrm{m}^{-2} . \mathrm{s}^{-1}\right)$ occurred between D10 and D14, corresponding to the end of the exponential phase (Figure 6a). One can here hypothesize that these metabolites could play a role in reducing the oxidative stress effects potentially induced by "higher light" condition [27].

On the other hand, other compounds present net intracellular concentration increases under "higher temperature" $\left(28^{\circ} \mathrm{C}\right)$ occurring during the early (from D14) or the late (from D24) parts of the stationary phase, depending on the molecule family, as exemplified with microginin 741A, microginin/cyanostatin A, microviridin K, microvidin 1724 and two dipeptides (Glu-Val and Glu-Cys) (Figure 6b). In contrast to light-induced metabolites, the concentrations of the temperature-induced metabolites appear to be less important and to increase only at the end of the exponential phase, or even latter at the end of the experiment. The difference of only $4^{\circ} \mathrm{C}$ that discriminates the "control" $\left(24^{\circ} \mathrm{C}\right)$ and "higher temperature" conditions $\left(28^{\circ} \mathrm{C}\right)$ therefore appears to have less general impact on the metabolite production and the cellular growing of Aliinostoc sp. PMC 882.14, than a light increase from 12 to $24 \mu \mathrm{mol} \cdot \mathrm{m}^{-2} \cdot \mathrm{s}^{-1}$, whereas the opposite results were described by Kumar et al. (2011) in Spirulina platensis with pigment production [28].

The strong increase in the concentrations of two MAAs (shinorine and mycosporineglycine) under high-light condition was consistent with the putative photo-protective role of MAAs against $U V$-B radiations [29]. However, the physiological function of MAA in cyanobacteria may be multiple and more generalist, as they may also act as anti-oxidants or osmo-protectors [30].

These variations in the intracellular metabolite relative contents can result from the summation of different cellular processes [31]. Thus, the variation in observed intracellular contents for a given metabolite can be either the consequence of $i$ ) a modulation of its biosynthesis rate depending of the physiological status of the cells, ii) a variation in its degradation rate, or iii) a change of its excretion rate by the cells through the extracellular compartment [32]; this latter supposition seems not to occur in the present case as none apparent metabolite transfer from the intra- to the extra-cellular compartment could have been revealed by the analyses.

Adenosine monophosphate (AMP) was a primary metabolite which exhibited in the present experiment higher relative concentrations during the stationary phase for both tested light and temperature conditions compared to the control (Figure 6). This accumulation could be the result of the degradation of ADP (adenosine diphosphate) and ATP (adenosine triphosphate), which may indicate an increase of energy consumption occurring under stress [33]. However, to confirm this hypothesis, it would be necessary to quantify ADP and ATP, as these latter compounds were not retrieved by the present un-targeted metabolomics pipeline based on variable extraction using differential analysis, in order to calculate the ratios between the amounts of AMP, ADP and ATP.

Furthermore, even if most metabolites belonging to the same family exhibit the same dynamics, some peptide variants seem to be produced preferentially to others depending on the culture conditions. This is remarkably the case for some microginin variants. Differences in the ratios of variants of secondary metabolites in other cyanobacteria have already been described, especially in the case of response to light stress [27]. In their study, Tonk and co-workers (2005) observed in Planktothrix agardhii a light-stress induced change in the ratio of the different variants of microcystins, cyanobacterial-specific secondary metabolites produced by NRPS/PKS complexes, similar to those responsible of microginin biosynthesis. The variations of the intracellular availability of the substrates necessary for the biosynthesis of these compounds could be an explanation for such differences. Indeed, microginins 755B, 741 and microginin/cyanostatin A structures differ in the composition of central amino acid (alanine, serine or valine, respectively), and the synthesis of one 
variant or another one can vary depending on the affinity of elongation enzymes to the substrates and of their respective availability[18].

\section{Materials and Methods}

\subsection{Biological material.}

Aliinostoc sp. PMC 882.14 strain was isolated in 2014 from the thermal mud of Balaruc-les-Bains (France) and kept in collection at the Paris Museum Collection (PMC, Paris. Four large volume $(500 \mathrm{~mL})$ of pre-cultures were cultivated in 1-L Durand's bottles up to 46 days in Z8 culture medium, with $12 \mu \mathrm{mol} . \mathrm{m}^{-2} . \mathrm{s}^{-1}$ light intensity and $12 \mathrm{~h}: 12 \mathrm{~h}$ lightdark photoperiod. Samples were collected after 33 days for chemical strain characterization. Pre-cultures bottles were thus pooled (on $46^{\text {th }}$ day) in order to obtain a large volume (above $2 \mathrm{~L}$ ) homogeneous stock solution (D0) that was further used for the experimentation. From this pool, the 1/10 diluted cultures were then inoculated in triplicats in 1-L bottles (initial volume of $500 \mathrm{~mL}$ ) for experimentations under "control", "higher light" and "higher temperature" conditions during the 28 following days. The Figure 1 represents the global experimental and analytical pipeline developed in this study.

\subsection{Culture conditions and sampling.}

Experiment was conducted over a 28-days period in "control" $\left(24^{\circ} \mathrm{C}, 12 \mu \mathrm{mol} . \mathrm{m}^{-2} . \mathrm{s}^{-}\right.$ $\left.{ }^{1}\right)$, “higher light" $\left(24^{\circ} \mathrm{C}, 24 \mu \mathrm{mol} \cdot \mathrm{m}^{-2} \cdot \mathrm{s}^{-1}\right)$ and "higher temperature" $\left(28^{\circ} \mathrm{C}, 12 \mu \mathrm{mol} . \mathrm{m}^{-2} \cdot \mathrm{s}^{-1}\right)$ conditions. Experimental conditions were performed in triplicated, under a $12 \mathrm{~h}: 12 \mathrm{~h}$ photoperiod and a constant homogenization maintained by magnetic agitators. Two weekly samples were taken throughout the 28 days (D0, D3, D7, D10, D14, D17, D21, D24, and D28). Each sampling (22 $\mathrm{mL})$ were sub-divided into several parts for $i$ ) the extraction and the analysis of intra and extra-cellular metabolites $(10 \mathrm{~mL})$, ii) the biomass monitoring using extraction and quantification of chlorophyll pigments $(10 \mathrm{~mL})$, optical density and cell counts $(2 \mathrm{~mL})$. Sampling at D0 was performed on the diluted $(1 / 10)$ stock solution, in triplicate.

\subsection{Biomass dynamics.}

Cyanobacterial biomass was followed using: absorbance at $750 \mathrm{~nm}$ by in vivo spectrophotometric (Cary 500; Varian, U.S.A.), chlorophyll- $a$ concentration [34] and cell abundance by Malassez' cell counts [35]. Data have been transformed into Neperian logarithm and statistical analysis between treatments and control (ANOVA with Bonferonni's correction) was conducted with GraphPad Prism 5.01 software.

\subsection{Metabolite extractions.}

Samples $(10 \mathrm{~mL})$ were centrifuged $\left(10 \mathrm{~min}, 3,220 \mathrm{~g}, 4^{\circ} \mathrm{C}\right)$. Supernatant was isolated and stored at $-20^{\circ} \mathrm{C}$ in prevision of extracellular metabolites liquid extractions. The pellet was centrifuged and stored at $-80^{\circ} \mathrm{C}$ for at least 24 hours, then the sample lyophilization was performed $\left(-50^{\circ} \mathrm{C}, 0.02 \mathrm{mBar}\right.$ for 16 hours) (Labconco, Italia). Dry weight was thus determined, and then the two sequential extractions performed (with $100 \mu \mathrm{L}$ of solvent for $1 \mathrm{mg}$ biomass dry weight). The first extraction of hydrophilic metabolites was carried out with a water/formic acid mixture $(0.1 \%)$, and a second extraction targeting hydrophobic metabolites was performed with a water/methanol mixture (75\%), then the two supernatants were pooled (apart for the pre-culture in order to compare metabolites composition in the three extraction fractions). For both extraction, cell lysis was performed by sonication: 3 cycles of $30 \mathrm{~s}, 10 \mathrm{~s}$ break between each cycle, at $80 \%$ of the maximum intensity (SONICS Vibra Cell, $130 \mathrm{Watt}, 20 \mathrm{Khz}$ ). Samples were then centrifuged (10 min, 13,400 g, $4^{\circ} \mathrm{C}$ ) and the supernatants collected and stored in obscurity at $-20^{\circ} \mathrm{C}$ before mass spectrometry analysis.

Extracellular metabolites present in the supernatant of the culture media (D0, D7, D14, D21, D28 as they corresponding to key moments of the growth phases) were extracted by liquid/liquid extraction with equivalent volume of solvent ( $100 \%$ ethyl acetate) with a separating funnel. Upper organic phase has been collected after homogenization 
and degassing. Water molecules in the organic phase were removed by the gradual addition of magnesium sulfate powder $\left(\mathrm{MgSO}_{4}\right)$. Consecutively to filtration, liquid was dried under air flow, then the dry extracts were re-suspended in a mixture of water/methanol $(75 \%)$ /formic acid $(0.1 \%)$ and centrifuged $\left(10 \mathrm{~min}, 13,400 \mathrm{~g}, 4^{\circ} \mathrm{C}\right)$ before storage at $-20^{\circ} \mathrm{C}$.

\subsection{Mass Spectrometry Analysis.}

Extracts were separated by ultra-high performance liquid chromatography (UHPLC). For each sample, $2 \mu \mathrm{L}$ was injected and molecules separation was performed by a Polar Advance II 2.5 pore C18 (Thermo) chromatographic column at a flow rate of $300 \mu \mathrm{L} . \mathrm{min}^{-1}$ under a linear gradient of acetonitrile acidified with $0.1 \%$ formic acid (from 5 to $90 \%$ in $15 \mathrm{~min}$ ). Metabolites contents were analyzed using an electrospray ionization hybrid quadrupole time-of-flight (ESI-QqTOF) high resolution mass spectrometer (Compact, Brucker) in the range $50-1500 \mathrm{~m} / \mathrm{z}$.

\subsection{Simple MS mode analyses.}

Compounds were initially analyzed in simple MS positive and negative modes, without quadrupole fragmentation. This mode of analysis notably allows observing a signal intensity of each ion (measured in number of detected counts over the time range necessary for its elution) which is directly proportional to the quantity of ions present in the different samples. This analysis was carried out in order to compare metabolites composition in the three extraction fractions on the pre-culture (representing a total 2,441 intracellular analytes extracted with acidified water or methanol and extracellular analytes, when data were merged), and in the samples collected from the experimentation (for either extra- or pooled intra-cellular fractions, analyzed on positive mode only). MS data were processed using MetaboScape 4.0 software (Bruker, Bremen, Germany) for recalibration of each sample analysis (according to internal standard), peak detection and selection of ions which intensity was greater than 5,000 counts in at least $10 \%$ of the set of samples and peak realigned. Furthermore, different states of charge and adducts were grouped together and the "area-under-the-peak" determined, in order to generate a unique global data matrix containing semi-quantification results for each metabolite, in all analyzed samples peak for each analyte (characterized by the respective mean mass of its neutral form and its corresponding retention time).

\subsection{MS/MS mode analyses.}

For qualitative investigation of metabolites, additional ion selection with the quadrupole and fragmentation was carried out in tandem by collision ion dissociation (CID) according to the autoMS/MS analysis, performed in positive mode. The ions with the highest intensities in single MS (MS1) were then selected by the quadrupole and fragmented in a collision cell. Resulting ions of the fragmentation of their respective parent were transferred and detected. This series of MS/MS dataset containing fragment list of all selected ions allow qualitatively the characterization of the diversity of the corresponding metabolites produced by Aliinostoc sp. PMC 882.14. through the comparison of the fragmentation profiles. The files containing the fragmentation information for each ion analyzed were exported in mgf format using MetaboScape 4.0 (Bruker) software, before being used for the generation of the molecular network of structural similarity.

Metabolites annotations were made from MS/MS data (mgf file) by generating a molecular network for the comparison of fragmentation profiles using MetGem software (version 1.1.2) and GNPS algorithm [36]. Public and generalist spectral databases GNPS library, NIH Clinical collections and EMBL metabolomics were used. Annotations were supplemented by a match with CyanoMetDB database, which contains all the raw formulae of the 2,100 cyanobacterial metabolites already described [25].

\subsection{Statistical treatments.}

MetaboAnalyst 4.0 platform [37] was used to perform data matrix normalization (Pareto), Principal Component Analysis (PCA), Partial Least Square Discriminant Analysis 
(PLS-DA), Multivariate Empirical Bayes Analysis (MEBA) [38] and ANOVA (ANalysis Of VAriance).

\section{Conclusions}

As a conclusion, this study provided a first picture of diversity and dynamics of metabolites produced by the cyanobacterium Aliinostoc sp. PMC 882.14, under different culture conditions. This strain was characterized by the presence of numerous BGCs in its genome, and the consecutive production of numerous and specific metabolites such as various analogues of somamides/dolastatins, microginins, microviridins and MAAs. The growth phases related to the physiological status of the cells appears to play a key role in the metabolic composition and abundance, with the most statistical variations occurring between exponential $v s$ stationary growth phases. Light and temperature also appear to have significant effects on the dynamic of the metabolic composition, and should be further considered, especially in the context of global metabolomic comparison between cyanobacteria and of bioactive compound production. Indeed, for valorization purposes the production of potentially bioactive components, such as shinorine, somamides/dolastatins and microginins, by Aliinostoc sp. PMC 882.14, represents great potential for further bioactivity screening taking into account culture conditions.

Supplementary Materials: The following are available online at www.mdpi.com/xxx/s1, Table S1: Biosynthetic gene clusters identified from Aliinostoc sp. PMC 882.14, Table S2: Metabolite annotations from MS/MS fragmentation data, Table S3: List of metabolites presenting the best MEBA scores (MB.statistics) with corresponding ANOVA analysis results. Variations over time course for metabolites highlighted in yellow are represented in Figure6. $\mathrm{MW}=$ Molecular Weight, $\mathrm{RT}=$ Retention Time.

Figure S1: Candidate microginin biosynthetic gene cluster from Aliinostoc sp. PMC 882.14,

Figure S2: Growth curves based on extracted chlorophyll $a$ and cell count. Significant differences between control and conditions are represented by a single star (ANOVA, pvalue $<0,05$ ) or two stars (ANOVA, pvalue $<0,01$ ).

Figure S3: Principal Component Analysis (PCA) representing the evolution of the intracellular metabolic composition of Aliinostoc sp. PMC 882.14 as a function of culture conditions (control= grey, "higher light" = yellow and "higher temperature"= red) a) PC1 and PC2 and b) PC1 and PC3.

Figure S4: PLS-DA considering the days of sampling for (a) control samples only and (b) samples from the three experimental conditions and (c,d) corresponding lists of the analytes contributing the most to the sample discrimination through the culture kinetics (variable of importance in the projection, VIP score >2). The red framed lines correspond to the metabolites in common with the analysis performed only on controls.

Figure S5: Box-plots representing the 29 intracellular molecules (exhibiting VIP scores > 2; Fig S4d), which explain the most of the differences between the different days of culture considering samples from the three experimental conditions

Figure S6: Box-plots representing the 38 intracellular molecules (exhibiting VIP scores > 2; Fig S4c), which explain the most of the differences between the different days of culture considering only control samples.

Figure S7: Principal Component Analysis (PCA) representing the evolution of the extracellular metabolic composition of Aliinostoc sp. PMC 882.14 as a function of number of culture days a) under the control condition, b) under control, "higher light" and "higher temperature" conditions. Each point represents a culture replicate. The green color gradient reflects the temporal scale of the cultures, 
Figure S8: Principal Component Analysis (PCA) representing the evolution of the extracellular metabolic composition of Aliinostoc sp. PMC 882.14 as a function of culture conditions (control= grey, "higher light" = yellow and "higher temperature"= red) a) PC1 and PC2 and b) PC1 and PC3.

Figure S9: PLS-DA representing the evolution of the extracellular metabolic composition of Aliinostoc sp. PMC 882.14 considering the days of sampling for (a) control samples only and (b) samples from the three experimental conditions and $(c, d)$ corresponding lists of the analytes contributing the most to the sample discrimination through the culture kinetics (variable of importance in the projection, VIP score >2). The red framed lines correspond to the metabolites in common with the analysis performed only on controls

Figure S10: Box-plots representing the 25 intracellular molecules (exhibiting VIP scores > 2; Fig S10d), which explain the most of the differences between the different days of culture considering samples from the three experimental conditions,

Author Contributions: Conceptualization, S.K.T, J.D. and B.M.; methodology, S.K.T, J.D. and B.M.; experiments D.L.M.; formal analysis, D.LM., J.D., S.K.T. and B.M.; writing-original draft preparation, D.L.M., J.D. and B.M.; writing-review and editing, D.L.M., J.D., S.K.T., A.R., C.B. and B.M. All authors have read and agreed to the published version of the manuscript.

Funding: This work was supported by the ANRT through a PhD grant awarded to J. Demay $\left(\mathrm{n}^{\circ} 2017 / 0633\right)$ and by ATM support provided by the MNHN.

Acknowledgments: We would like to thank the UMR 7245 MCAM, Muséum National d'Histoire Naturelle, Paris, France for laboratories facilities and the Thermes de Balaruc-les-Bains for founds. This work was supported by the "Société Publique Locale d'Exploitation des Thermes de Balarucles-Bains (SPLETH), the town of Balaruc-les-Bains and the National Muséum of Natural History.

Conflicts of Interest: The authors declare no conflict of interest.

\section{References}

1. Whitton, B.A.; Potts, M. Ecology of Cyanobacteria II: Their Diversity in Space and Time. Kluwer Academic Publishers: Dordrecht 2012, pp. 1-13.

2. Dittmann, E.; Gugger, M.; Sivonen, K.; Fewer, D.P. Natural Product Biosynthetic Diversity and Comparative Genomics of the Cyanobacteria. Trends Microbiol. 2015, 23, 642-652.

3. Leão, P.N.; Engene, N.; Antunes, A.; Gerwick, W.H.; Vasconcelos, V. The chemical ecology of cyanobacteria. Nat. Prod. Rep. $2012,29,372-391$.

4. Gerwick, W.H.; Fenner, A.M. Drug Discovery from Marine Microbes. Microb Ecol 2013, 800-806.

5. Singh, R.; Parihar, P.; Singh, M.; Bajguz, A.; Kumar, J.; Singh, S.; Singh, V.P.; Prasad, S.M. Uncovering potential applications of cyanobacteria and algal metabolites in biology, agriculture and medicine: Current status and future prospects. Front. Microbiol. 2017, 8, 515.

6. Rastogi, R.P.; Sonani, R.R.; Madamwar, D. Cyanobacterial Sunscreen Scytonemin: Role in Photoprotection and Biomedical Research. Appl. Biochem. Biotechnol. 2015, 176, 1551-1563.

7. Lawrence, K.P.; Gacesa, R.; Long, P.F.; Young, A.R. Molecular photoprotection of human keratinocytes in vitro by the naturally occurring mycosporine-like amino acid palythine. Br. J. Dermatol. 2018, 178, 1353-1363.

8. Welker, M.; Von Döhren, H. Cyanobacterial peptides - Nature's own combinatorial biosynthesis. FEMS Microbiol. Rev. 2006, 30, 530-563.

9. Kehr, J.C.; Picchi, D.G.; Dittmann, E. Natural product biosyntheses in cyanobacteria: A treasure trove of unique enzymes. Beilstein J. Org. Chem. 2011, 7, 1622-1635.

10. Demay, J.; Bernard, C.; Reinhardt, A.; Marie, B. Natural products from cyanobacteria: Focus on beneficial activities. Mar. Drugs 2019, 17, 320. 
11. Holland, A.; Kinnear, S. Interpreting the Possible Ecological Role(s) of Cyanotoxins: Compounds for Competitive Advantage and/or Physiological Aide? Mar. Drugs 2013, 11, 2239-2258.

12. Leflaive, J.; Ten-Hage, L. Algal and cyanobacterial secondary metabolites in freshwaters: A comparison of allelopathic compounds and toxins. Freshw. Biol. 2007, 52, 199-214.

13. Kameyama, K.; Sugiura, N.; Inamori, Y.; Maekawa, T. Characteristics of microcystin production in the cell cycle ofMicrocystis viridis. Environ. Toxicol. 2004, 19, 20-25.

14. Natumi, R.; Janssen, E.M.L. Cyanopeptide Co-Production Dynamics beyond Mirocystins and Effects of Growth Stages and Nutrient Availability. Environ. Sci. Technol. 2020, 54, 6063-6072.

15. Barofsky, A.; Vidoudez, C.; Pohnert, G. Metabolic profiling reveals growth stage variability in diatom exudates. Limnol. Oceanogr. Methods 2009, 7, 382-390.

16. Jahn, M.; Vialas, V.; Karlsen, J.; Maddalo, G.; Edfors, F.; Forsström, B.; Uhlén, M.; Käll, L.; Hudson, E.P. Growth of Cyanobacteria Is Constrained by the Abundance of Light and Carbon Assimilation Proteins. Cell Rep. 2018, 25, 478-486.e8.

17. Paerl, H.W.; Otten, T.G. Harmful Cyanobacterial Blooms: Causes, Consequences, and Controls. Microb. Ecol. 2013, 65, 9951010.

18. Davis, T.W.; Berry, D.L.; Boyer, G.L.; Gobler, C.J. The effects of temperature and nutrients on the growth and dynamics of toxic and non-toxic strains of Microcystis during cyanobacteria blooms. Harmful Algae 2009, 8, 715-725.

19. Jain, S.; Prajapat, G.; Abrar, M.; Ledwani, L.; Singh, A.; Agrawal, A. Cyanobacteria as efficient producers of mycosporine-like amino acids. J. Basic Microbiol. 2017, 57, 715-727.

20. Duval, C.; Hamlaoui, S.; Piquet, B.; Toutirais, G.; Yéprémian, C.; Reinhardt, A.; Duperron, S.; Marie, B.; Demay, J.; Bernard, C. Diversity of cyanobacteria from thermal muds (Balaruc-Les-Bains, France) with the description of Pseudochroococcus coutei gen. nov., sp. nov. FEMS Microbes 2021, 2, 6.

21. Demay, J.; Halary, S.; Knittel-Obrecht, A.; Villa, P.; Duval, C.; Hamlaoui, S.; Roussel, T.; Yéprémian, C.; Reinhardt, A.; Bernard, C.; et al. Anti-Inflammatory, Antioxidant, and Wound-Healing Properties of Cyanobacteria from Thermal Mud of BalarucLes-Bains, France: A Multi-Approach Study. Biomolecules 2020, 11, 28.

22. Nogle, L.M.; Williamson, R.T.; Gerwick, W.H. Somamides A and B, two new depsipeptide analogues of dolastatin 13 from a Fijian cyanobacterial assemblage of Lyngbya majuscula and Schizothrix species. J. Nat. Prod. 2001, 64, 716-719.

23. Zervou, S.K.; Gkelis, S.; Kaloudis, T.; Hiskia, A.; Mazur-Marzec, H. New microginins from cyanobacteria of Greek freshwaters. Chemosphere 2020, 248, 125961.

24. Süssmuth, R.D.; Mainz, A. Nonribosomal Peptide Synthesis-Principles and Prospects. Angew. Chemie - Int. Ed. 2017, 56, 3770-3821.

25. Jones, M.R.; Pinto, E.; Torres, M.A.; Dörr, F.; Mazur-Marzec, H.; Szubert, K.; Tartaglione, L.; Dell'Aversano, C.; Miles, C.O.; Beach, D.G.; et al. Comprehensive database of secondary metabolites from cyanobacteria. bioRxiv 2020, 2020.04.16.038703.

26. Tikhonova, I.; Kuzmin, A.; Deeva, D.; Sorokovikova, E.; Potapov, S.; Lomakina, A.; Belykh, O. Cyanobacteria Nostoc Punctiforme from Abyssal Benthos of Lake Baikal: Unique Ecology and Metabolic Potential. Indian J. Microbiol. 2017, 57, 422426.

27. Tonk, L.; Visser, P.M.; Christiansen, G.; Dittmann, E.; Snelder, E.O.F.M.; Wiedner, C.; Mur, L.R.; Huisman, J. The microcystin composition of the cyanobacterium Planktothrix agardhii changes toward a more toxic variant with increasing light intensity. Appl. Environ. Microbiol. 2005, 71, 5177-5181.

28. Kumar, M.; Kulshreshtha, J.; Singh, G.P. Growth and biopigment accumulation of cyanobacterium Spirulina platensis at different light intensities and temperature. Brazilian J. Microbiol. 2011, 42, 1128-1135.

29. Řezanka, T.; Temina, M.; Tolstikov, A.G.; Dembitsky, V.M. Natural microbial UV radiation filters - Mycosporine-like amino acids. Folia Microbiol. (Praha). 2004, 49, 339-352. 
30. Kageyama, H.; Waditee-Sirisattha, R. Mycosporine-Like Amino Acids as Multifunctional Secondary Metabolites in Cyanobacteria: From Biochemical to Application Aspects; 1st ed.; Elsevier B.V., 2018; Vol. 59; ISBN 9780444641793.

31. Kultschar, B.; Dudley, E.; Wilson, S.; Llewellyn, C.A. Intracellular and Extracellular Metabolites from the Cyanobacterium Chlorogloeopsis fritschii, PCC 6912, During 48 Hours of UV-B Exposure. Metab. 2019, Vol. 9, Page 74 2019, 9, 74.

32. Pinu, F.R.; Granucci, N.; Daniell, J.; Han, T.-L.; Carneiro, S.; Rocha, I.; Nielsen, J.; Villas-Boas, S.G. Metabolite secretion in microorganisms: the theory of metabolic overflow put to the test. Metabolomics 2018144 2018, 14, 1-16.

33. Cano, M.; Holland, S.C.; Artier, J.; Burnap, R.L.; Ghirardi, M.; Morgan, J.A.; Yu, J. Glycogen Synthesis and Metabolite Overflow Contribute to Energy Balancing in Cyanobacteria. Cell Rep. 2018, 23, 667-672.

34. Yéprémian, C.; Catherine, A.; Bernard, C.; Congestri, R.; Elersek, T.; Pilkaityte, R. Chlorophyll a Extraction and Determination. In Handbook of Cyanobacterial Monitoring and Cyanotoxin Analysis; John Wiley \& Sons, Ltd: Chichester, UK, 2017; pp. 331-334.

35. Catherine, A.; Maloufi, S.; Congestri, R.; Viaggiu, E.; Pilkaityte, R. Cyanobacterial samples: preservation, enumeration, and biovolume measurements. In Handbook of Cyanobacterial Monitoring and Cyanotoxin Analysis; Wiley, J., Ed.; 2017; pp. 315-330.

36. Wang, M.; Carver, J.J.; Phelan, V. V.; Sanchez, L.M.; Garg, N.; Peng, Y.; Nguyen, D.D.; Watrous, J.; Kapono, C.A.; LuzzattoKnaan, T.; et al. Sharing and community curation of mass spectrometry data with Global Natural Products Social Molecular Networking. Nat. Biotechnol. 2016, 34, 828-837.

37. Chong, J.; Wishart, D.S.; Xia, J. Using MetaboAnalyst 4.0 for Comprehensive and Integrative Metabolomics Data Analysis. Curr. Protoc. Bioinforma. 2019, 68.

38. Tai, Y.C.; Speed, T.P. A multivariate empirical Bayes statistic for replicated microarray time course data. Ann. Stat. 2006, 34, 2387-2412. 